\title{
67. COMPRESSIONAL WAVE VELOCITIES, DENSITIES, AND POROSITIES OF BASALTS FROM HOLES 417A, 417D, AND 418A, DEEP SEA DRILLING PROJECT LEGS 51-53
}

\author{
N.I. Christensen, ${ }^{1}$ S.C. Blair, ${ }^{2}$ R.H. Wilkens,${ }^{3}$ and M.H. Salisbury ${ }^{4}$
}

\section{INTRODUCTION}

It has long been suspected that the seismic velocity of at least the upper level of Layer 2 is controlled not only by the intrinsic velocity of the rocks of which it is composed, but by the presence of water-filled cracks and voids. By comparing laboratory measurements of compressional wave velocity through basalts recovered from Hole $417 \mathrm{D}$, with the results of the oblique seismic experiment (Stephen et al., this volume) and logging (Salisbury et al., this volume) for the same hole, it should be possible for the first time to evaluate the role of such cracks upon velocity in the upper few hundred meters of the oceanic crust. Since seismic velocities are extremely sensitive to confining pressure (e.g., Birch, 1960) any such evaluation must be based on laboratory measurements of velocity at elevated pressures appropriate in situ conditions.

In this paper, we present compressional wave velocities to hydrostatic confining pressures of $6 \mathrm{kbar}$ for 88 samples of basalt from Holes 417A, 417D, and 418A. In addition, bulk densities and porosities have been measured and are shown to correlate well with the compressional wave velocities. Of particular significance, basalt velocities from these sites are shown to decrease linearly with increasing porosity.

\section{EXPERIMENTAL TECHNIQUES AND DATA}

Prior to velocity measurement, each sample was cut to a right cylinder ( $2.5 \mathrm{~cm}$ in diameter and 3 to $5 \mathrm{~cm}$ in length) and then air-dried, measured, and weighed to determine its dry-bulk density. The samples were then water-saturated and reweighed to obtain wet-bulk densities and effective porosities. After weighing, the saturated samples were jacketed with copper foil and 1-MHz barium titanate transducers were attached to the core ends. The compressional wave velocity through each sample was then determined using the pulse transmission technique of Birch (1960). Pore pressures were maintained at values lower than external pressures by placing 100 mesh screen between the samples and copper jackets. Pressure was applied to the samples by means of a two-stage intensifier with low viscosity oil as the pressure medium. Pressure was measured using a calibrated manganin coil.

\footnotetext{
'Department of Geological Sciences, Graduate Program in Geophysics and Department of Oceanography, University of Washington, Seattle, Washington.

${ }^{2}$ Graduate Program in Geophysics, University of Washington, Seattle, Washington.

${ }^{3}$ Department of Geological Sciences, University of Washington, Seattle, Washington.

${ }^{4}$ Scripps Institution of Oceanography, University of California, La Jolla, California.
}

Velocities at 0.2 -kbar intervals to $1 \mathrm{kbar}$ and at larger intervals to $6 \mathrm{kbar}$, together with wet-bulk densities and effective porosities at atmospheric pressure are given in Table 1 . Above $1 \mathrm{kbar}$, the velocities are accurate to within 1 per cent (Christensen and Shaw, 1970). At lower pressures, the velocities can only be estimated to \pm 3 per cent because of signal attenuation.

\section{VELOCITY-DENSITY RELATIONS}

Previous investigations have demonstrated an excellent correlation between compressional wave velocity and bulk density in rocks under confining pressures to $10 \mathrm{kbar}$ (e.g., Birch, 1960; Christensen and Salisbury, 1975). It is desirable to formulate velocity-density correlations so that seismic velocities observed in situ may be used to determine the distribution of density with depth.

Compressional wave velocities for the 88 basaltic rocks studied from Holes 417A, 417D, and 418A range from 3.11 to $6.68 \mathrm{~km} / \mathrm{s}$ and wet bulk densities range from 2.10 to 2.96 $\mathrm{g} / \mathrm{cm}^{3}$. Figure 1 presents the compressional wave velocity $\left(V_{p}\right)$ at 1 kbar versus wet bulk density $(\rho)$ for these samples. Included in Figure 1 are linear and parabolic regressions of velocity versus density for 77 DSDP basalts studied by Christensen and Salisbury (1975) with parameters:

$$
\begin{gathered}
V_{p}=4.10+3.52 \rho \\
V_{p}=2.37+0.85 \rho^{3.57}
\end{gathered}
$$

Data from the current samples usually fall within \pm 0.2 $\mathrm{km} / \mathrm{s}$ of the non linear predictor except for the sample from Section 417D-28-5, a greenstone $\left(\rho=2.68 \mathrm{~g} / \mathrm{cm}^{3}\right.$ and $\mathrm{V}_{\mathrm{p}}=5.86 \mathrm{~km} / \mathrm{s}$ ) which falls above the basaltic trends. Table 2 includes linear regression parameters for $V_{p}$ versus $\rho$ using values of $\mathrm{V}_{\mathrm{p}}$ measured at $0.2,1$, and $6 \mathrm{kbar}$ for 88 samples.

Many samples from Hole 418A that were cored at depths between 710 and 862 meters sub-bottom exhibit densities greater than $2.85 \mathrm{~g} / \mathrm{cm}^{3}$ and account for most of the high density points in Figure 1. Samples from Hole 417D, on the other hand, were recovered from depths between 345 and 696 meters sub-bottom and have moderate densities; basalts cored from between 218 and 412 meters in Hole 417A range in density from $2.90 \mathrm{~g} / \mathrm{cm}^{3}$ to values as low as $2.10 \mathrm{~g} / \mathrm{cm}^{3}$ near the top of the basement. Thus, a general increase in density with sub-bottom depth is observed. Examination of the cored samples suggests that this increase in density with depth corresponds to a decrease in alteration.

\section{VELOCITY/POROSITY RELATIONSHIPS}

Figure 2 represents a plot of compressional wave velocity at $1 \mathrm{kbar}$ versus effective porosity. Correlation between the 
TABLE 1

Velocities, Densities, and Porosities

\begin{tabular}{|c|c|c|c|c|c|c|c|c|}
\hline Sample & $\begin{array}{c}\text { Bulk } \\
\text { Density }\end{array}$ & & & & $\begin{array}{l}\text { ores } \\
\text { at }\end{array}$ & $\begin{array}{l}\text { nal } \\
\text { ying }\end{array}$ & $\begin{array}{l}\text { Vel } \\
\text { ssure }\end{array}$ & $\begin{array}{l}\text { ty (km } \\
\text { sbar) }\end{array}$ \\
\hline (Interval in cm) & $\left(\mathrm{g} / \mathrm{cm}^{3}\right)$ & (9) & 0.2 & 0.4 & 0.6 & 0.8 & 1.0 & 2.0 \\
\hline $417 \mathrm{~A}-24-1,115-117$ & 2.451 & 11.3 & 3.82 & 3.86 & 3.89 & 3.92 & 3.95 & 4.08 \\
\hline 417 A- $25-1,126-128$ & 2.569 & 8.6 & 4.72 & 4.75 & 4.78 & 4.81 & 4.83 & 4.91 \\
\hline $417 \mathrm{~A}-27-1,49-51$ & 2.692 & 3.7 & 5.25 & 5.32 & 5.36 & 5.39 & 5.41 & 5.50 \\
\hline $417 \mathrm{~A}-28-6,71-73$ & 2.732 & 2.6 & 5.30 & 5.36 & 5.40 & 5.44 & 5.48 & 5.60 \\
\hline $417 \mathrm{~A}-30-4,64-67$ & 2.763 & 2.5 & 5.52 & 5.57 & 5.59 & 5.62 & 5.64 & 5.71 \\
\hline $417 \mathrm{~A}-31 \cdot 3,26-29$ & 2.103 & - & 2.98 & 3.02 & 3.05 & 3.08 & 3.11 & 3.25 \\
\hline $417 \mathrm{~A}-32-4,20-23$ & 2.621 & 4.4 & 5.38 & 5.41 & 5.44 & 5.46 & 5.48 & 5.56 \\
\hline $417 \mathrm{~A}-34-3,135-138$ & 2.756 & 3.2 & 5.68 & 5.70 & 5.71 & 5.73 & 5.74 & 5.78 \\
\hline $417 \mathrm{~A}-37-1,57-60$ & 2.817 & 2.0 & 6.00 & 6.02 & 6.03 & 6.05 & 6.06 & 6.11 \\
\hline $417 \mathrm{~A}-38-4,10-12$ & 2.721 & 3.4 & 5.43 & 5.49 & 5.53 & 5.55 & 5.57 & 5.63 \\
\hline $417 \mathrm{~A}-42-6,4-6$ & 2.698 & 3.9 & 5.13 & 5.18 & 5.22 & 5.24 & 5.27 & 5.36 \\
\hline $417 \mathrm{~A}-44-3,68-70$ & 2.900 & 1.0 & 6.14 & 6.17 & 6.20 & 6.23 & 6.25 & 6.32 \\
\hline $417 \mathrm{~A}-46-4,43-45$ & 2.675 & 2.4 & 5.32 & 5.35 & 5.38 & 5.40 & 5.43 & 5.52 \\
\hline $417 \mathrm{D}-22-1,144-146$ & 2.708 & 5.4 & 5.13 & 5.16 & 5.18 & 5.20 & 5.21 & 5.29 \\
\hline $417 \mathrm{D}-22-6,110-112$ & 2.774 & 3.8 & 5.38 & 5.42 & 5.45 & 5.48 & 5.50 & 5.58 \\
\hline $417 \mathrm{D}-26-1,94-96$ & 2.776 & 3.3 & 5.35 & 5.41 & 5.46 & 5.50 & 5.53 & 5.63 \\
\hline $417 \mathrm{D}-27-4,34-36$ & 2.805 & 3.4 & 5.56 & 5.60 & 5.62 & 5.64 & 5.66 & 5.73 \\
\hline $417 D-28-2,49-51$ & 2.726 & 3.7 & 5.17 & 5.24 & 5.29 & 5.33 & 5.37 & 5.52 \\
\hline $417 \mathrm{D}-28-7,33-35$ & 2.677 & 3.8 & 5.78 & 5.81 & 5.83 & 5.87 & 5.88 & 5.94 \\
\hline $417 \mathrm{D}-29-1,84-86$ & 2.703 & 5.0 & 5.16 & 5.18 & 5.21 & 5.23 & 5.25 & 5.33 \\
\hline 417D-30-1, 118-120 & 2.822 & 1.7 & 5.78 & 5.84 & 5.87 & 5.91 & 5.94 & 6.04 \\
\hline $417 D-31-1,144-146$ & 2.737 & 3.0 & 5.26 & 5.30 & 5.34 & 5.37 & 5.39 & 5.48 \\
\hline $417 \mathrm{D}-32-5,120-122$ & 2.897 & 0.9 & 6.02 & 6.06 & 6.09 & 6.11 & 6.13 & 6.20 \\
\hline $417 \mathrm{D}-33-2,141-143$ & 2.835 & 1.5 & 6.01 & 6.11 & 6.17 & 6.21 & 6.25 & 6.34 \\
\hline $417 \mathrm{D}-34-2,55-57$ & 2.863 & 1.2 & 5.92 & 5.98 & 6.02 & 6.06 & 6.09 & 6.22 \\
\hline $417 \mathrm{D}-35-2,46-48$ & 2.752 & 3.6 & 5.58 & 5.62 & 5.66 & 5.68 & 5.70 & 5.77 \\
\hline $417 \mathrm{D}-37-2,59-61$ & 2.799 & 2.8 & 5.58 & 5.61 & 5.65 & 5.68 & 5.71 & 5.82 \\
\hline $417 \mathrm{D}-39-1,130-132$ & 2.360 & 8.7 & 3.80 & 3.91 & 3.99 & 4.05 & 4.10 & 4.24 \\
\hline $417 D-39-4,90-92$ & 2.855 & 1.9 & 5.95 & 6.02 & 6.08 & 6.11 & 6.15 & 6.28 \\
\hline $417 \mathrm{D}-40-1,87-89$ & 2.773 & 2.5 & 5.75 & 5.80 & 5.84 & 5.88 & 5.91 & 6.03 \\
\hline $417 \mathrm{D}-42-2,139-141$ & 2.669 & 5.1 & 5.33 & 5.38 & 5.41 & 5.44 & 5.46 & 5.53 \\
\hline $417 D-43-2,110-112$ & 2.811 & 3.0 & 5.70 & 5.73 & 5.75 & 5.76 & 5.78 & 5.85 \\
\hline $417 \mathrm{D}-44-1,142-144$ & 2.810 & 4.6 & 5.56 & 5.59 & 5.62 & 5.65 & 5.66 & 5.73 \\
\hline $417 \mathrm{D}-45-2,29-31$ & 2.796 & 4.5 & 5.50 & 5.54 & 5.58 & 5.60 & 5.62 & 5.69 \\
\hline $417 \mathrm{D}-48-6,50-53$ & 2.750 & - & 5.22 & 5.27 & 5.31 & 5.34 & 5.37 & 5.48 \\
\hline $417 \mathrm{D}-50-2,83-85$ & 2.830 & 2.5 & 5.71 & 5.74 & 5.78 & 5.81 & 5.83 & 5.92 \\
\hline $417 \mathrm{D}-52-2,123-126$ & 2.927 & 0.9 & 6.06 & 6.13 & 6.19 & 6.24 & 6.29 & 6.45 \\
\hline 417D-55-4, 43-46 & 2.851 & 2.6 & 5.80 & 5.84 & 5.88 & 5.92 & 5.95 & 6.06 \\
\hline $417 \mathrm{D}-58-3,99-101$ & 2.672 & 6.9 & 4.97 & 5.05 & 5.13 & 5.18 & 5.23 & 5.37 \\
\hline $417 \mathrm{D}-60-2,37-39$ & 2.769 & 4.2 & 5.50 & 5.55 & 5.58 & 5.60 & 5.63 & 5.72 \\
\hline $417 \mathrm{D}-62-1,12-15$ & 2.738 & 5.4 & 5.36 & 5.44 & 5.50 & 5.55 & 5.58 & 5.72 \\
\hline $417 \mathrm{D}-66-3,35-38$ & 2.895 & 1.6 & 6.08 & 6.10 & 6.12 & 6.13 & 6.15 & 6.22 \\
\hline $417 \mathrm{D}-67-4,48-50$ & 2.818 & 2.8 & 5.73 & 5.79 & 5.83 & 5.87 & 5.90 & 5.99 \\
\hline $418 \mathrm{~A}-15-1,28-30$ & 2.728 & 1.6 & 5.38 & 5.41 & 5.44 & 5.46 & 5.48 & 5.58 \\
\hline $418 \mathrm{~A}-16-3,44-47$ & 2.613 & 5.6 & 4.73 & 4.79 & 4.83 & 4.87 & 4.90 & 5.01 \\
\hline $418 \mathrm{~A}-18-1,47-50$ & 2.864 & 1.2 & 6.06 & 6.11 & 6.14 & 6.17 & 6.20 & 6.30 \\
\hline $418 \mathrm{~A}-20-2,94-96$ & 2.903 & 0.5 & 6.27 & 6.32 & 6.36 & 6.39 & 6.41 & 6.49 \\
\hline $418 \mathrm{~A}-25-1,145-147$ & 2.802 & 2.2 & 5.57 & 5.62 & 5.66 & 5.69 & 5.72 & 5.82 \\
\hline $418 \mathrm{~A}-26-3,21-24$ & 2.716 & 2.8 & 5.21 & 5.25 & 5.28 & 5.31 & 5.33 & 5.42 \\
\hline $418 \mathrm{~A}-28-1,130-132$ & 2.714 & 1.4 & 5.38 & 5.45 & 5.50 & 5.53 & 5.56 & 5.66 \\
\hline $418 \mathrm{~A}-30-4,23-26$ & 2.696 & 1.9 & 5.47 & 5.53 & 5.56 & 5.59 & 5.61 & 5.69 \\
\hline $418 \mathrm{~A}-33-1,79-81$ & 2.829 & 0.7 & 5.91 & 5.95 & 5.98 & 6.01 & 6.03 & 6.10 \\
\hline $418 \mathrm{~A}-35-1,28-30$ & 2.663 & 3.5 & 5.09 & 5.11 & 5.13 & 5.16 & 5.17 & 5.27 \\
\hline $418 \mathrm{~A}-35-5,22-24$ & 2.532 & 5.0 & 4.62 & 4.65 & 4.68 & 4.70 & 4.72 & 4.82 \\
\hline $418 \mathrm{~A}-39-2,40-42$ & 2.802 & 0.8 & 5.81 & 5.84 & 5.87 & 5.89 & 5.91 & 5.98 \\
\hline $418 \mathrm{~A}-41-1,94-96$ & 2.800 & 3.8 & 5.60 & 5.64 & 5.66 & 5.68 & 5.70 & 5.78 \\
\hline $418 \mathrm{~A}-41-3,62-65$ & 2.359 & 9.7 & 3.98 & 4.12 & 4.24 & 4.35 & 4.44 & 4.65 \\
\hline $418 \mathrm{~A}-42-4,63-65$ & 2.787 & 0.6 & 5.60 & 5.64 & 5.67 & 5.69 & 5.71 & 5.78 \\
\hline $418 \mathrm{~A}-44-1,43-46$ & 2.792 & 0.9 & 5.83 & 5.87 & 5.90 & 5.93 & 5.96 & 6.03 \\
\hline $418 \mathrm{~A}-46-1,10-12$ & 2.800 & 1.0 & 5.83 & 5.86 & 5.89 & 5.91 & 5.93 & 6.02 \\
\hline $418 \mathrm{~A}-48-1,26-28$ & 2.794 & 0.7 & 5.90 & 5.95 & 5.98 & 6.01 & 6.03 & 6.10 \\
\hline $418 \mathrm{~A}-49-2,102-104$ & 2.811 & 1.0 & 5.95 & 5.96 & 5.98 & 5.99 & 6.00 & 6.06 \\
\hline $418 \mathrm{~A}-51-2,100-102$ & 2.813 & 1.2 & 5.73 & 5.78 & 5.83 & 5.86 & 5.90 & 6.01 \\
\hline $418 \mathrm{~A}-52-3,132-134$ & 2.844 & 2.2 & 5.81 & 5.86 & 5.90 & 5.93 & 5.96 & 6.07 \\
\hline $418 \mathrm{~A}-54-2,7-9$ & 2.919 & 0.9 & 6.08 & 6.14 & 6.18 & 6.21 & 6.24 & 6.32 \\
\hline $418 \mathrm{~A}-55-2,2-4$ & 2.908 & 2.3 & 5.94 & 5.98 & 6.02 & 6.05 & 6.07 & 6.16 \\
\hline $418 \mathrm{~A}-56-1,110-112$ & 2.861 & 1.2 & 5.99 & 6.02 & 6.05 & 6.07 & 6.09 & 6.19 \\
\hline $418 \mathrm{~A}-57-2,122-124$ & 2.854 & 0.9 & 5.87 & 5.89 & 5.90 & 5.92 & 5.93 & 6.00 \\
\hline $418 \mathrm{~A} \cdot 60-6,21-23$ & 2.870 & 0.7 & 6.12 & 6.13 & 6.15 & 6.16 & 6.18 & 6.24 \\
\hline $418 \mathrm{~A}-61-1,5-7$ & 2.903 & 0.6 & 5.97 & 6.02 & 6.06 & 6.09 & 6.12 & 6.22 \\
\hline $418 \mathrm{~A}-63-5,44-46$ & 2.833 & 3.3 & 5.71 & 5.75 & 5.79 & 5.83 & 5.86 & 5.97 \\
\hline $418 \mathrm{~A}-64-1,89-91$ & 2.810 & 3.0 & 5.75 & 5.80 & 5.84 & 5.87 & 5.90 & 6.03 \\
\hline $418 \mathrm{~A}-65-1,63-65$ & 2.812 & 3.0 & 5.52 & 5.57 & 5.59 & 5.62 & 5,65 & 5.74 \\
\hline $418 \mathrm{~A}-67-1,40-42$ & 2.929 & 1.2 & 6.07 & 6.13 & 6.17 & 6.21 & 6.24 & 6.36 \\
\hline $418 \mathrm{~A}-69-1,95-97$ & 2.912 & 0.9 & 6.17 & 6.22 & 6.26 & 6.28 & 6.31 & 6.40 \\
\hline $418 \mathrm{~A}-71-1,18-20$ & 2.917 & 1.2 & 6.17 & 6.20 & 6.23 & 6.26 & 6.28 & 6.35 \\
\hline $418 \mathrm{~A}-73-1,106-108$ & 2.935 & 0.4 & 6.31 & 6.36 & 6.39 & 6.42 & 6.44 & 6.53 \\
\hline $418 \mathrm{~A}-74-1,98-100$ & 2.940 & 0.5 & 6.39 & 6.40 & 6.42 & 6.43 & 6.45 & 6.50 \\
\hline $418 \mathrm{~A}-77-5,26-28$ & 2.869 & 1.1 & 6.08 & 6.11 & 6.14 & 6.17 & 6.19 & 6.27 \\
\hline $418 \mathrm{~A}-78-6,65-67$ & 2.904 & 0.6 & 6.26 & 6.30 & 6.33 & 6.36 & 6.38 & 6.45 \\
\hline $418 \mathrm{~A}-79-3,67-69$ & 2.920 & 0.8 & 6.39 & 6.42 & 6.45 & 6.48 & 6.49 & 6.56 \\
\hline $418 \mathrm{~A}-80-2,68-70$ & 2.921 & 0.5 & 6.28 & 6.31 & 6.34 & 6.36 & 6.38 & 6.46 \\
\hline $418 \mathrm{~A}-81-5,110-112$ & 2.935 & 0.4 & 6.40 & 6.45 & 6.48 & 6.50 & 6.53 & 6.60 \\
\hline $418 \mathrm{~A}-82-1,125-127$ & 2.929 & 0.0 & 6.28 & 6.33 & 6.37 & 6.40 & 6.43 & 6.55 \\
\hline $418 \mathrm{~A}-83 \cdot 2,56-58$ & 2.916 & 0.2 & 6.39 & 6.50 & 6.56 & 6.61 & 6.64 & 6.76 \\
\hline $418 \mathrm{~A} \cdot 84-2,22-24$ & 2.907 & 1.0 & 6.25 & 6.30 & 6.33 & 6.36 & 6.38 & 6.48 \\
\hline $418 \mathrm{~A}-85 \cdot 2,7-9$ & 2.955 & 0.1 & 6.47 & 6.56 & 6.61 & 6.65 & 6.68 & 6.80 \\
\hline $418 \mathrm{~A}-86-2,71-73$ & 2.876 & 1.7 & 5.99 & 6.03 & 6.06 & 6.08 & 6.10 & 6.18 \\
\hline
\end{tabular}




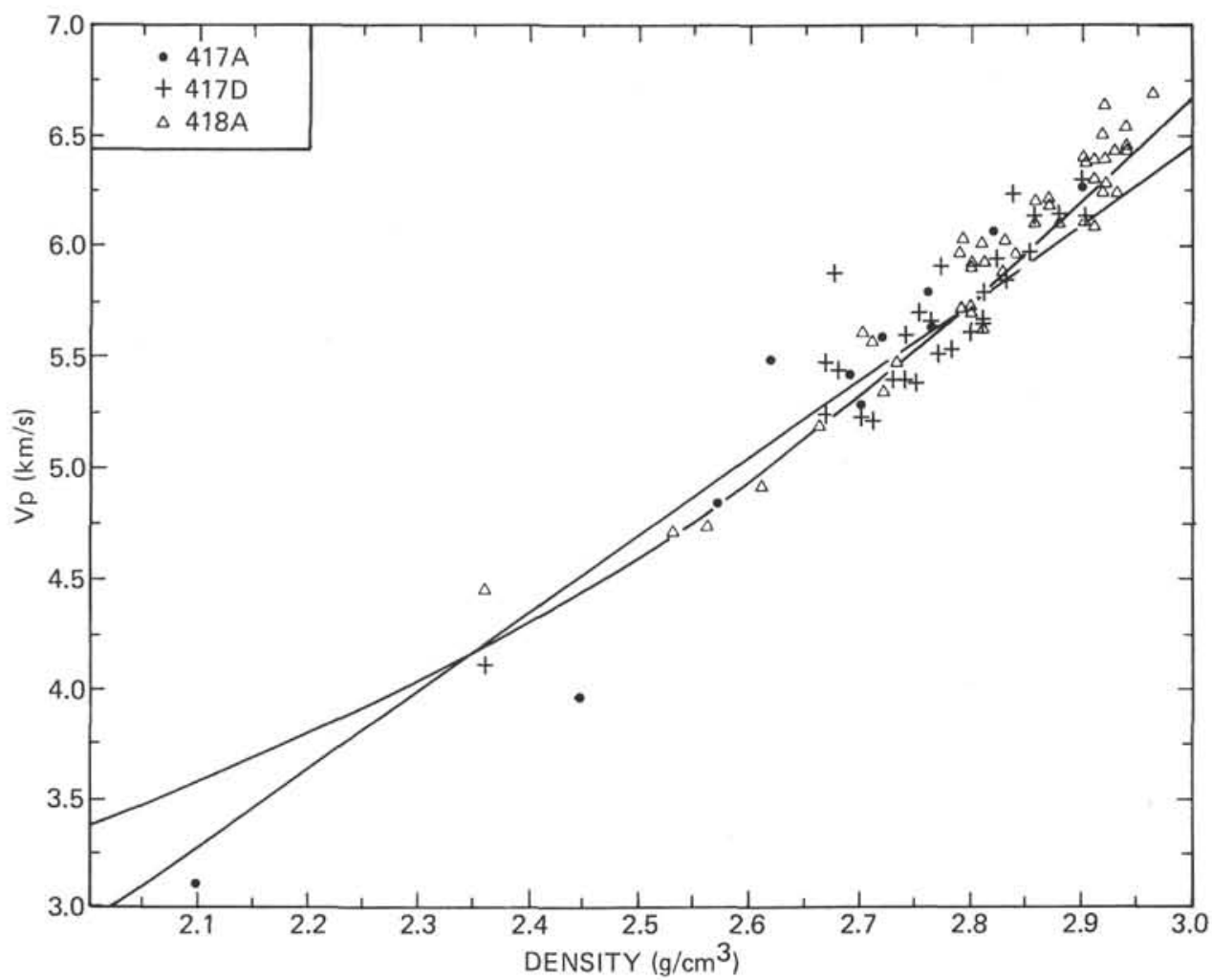

Figure 1. Compressional wave velocity at $1.0 \mathrm{kbar}$ plotted against wet-bulk density. Linear and non-linear solutions from Christensen and Salisbury (1975).

two parameters is quite good, not only at $1 \mathrm{kbar}$, as illustrated, but also at 0.2 and $6.0 \mathrm{kbar}$. Statistics and regression parameters are given for all three correlations in Table 2. The correlation is even more remarkable given the low accuracy of the porosity determination $( \pm 0.5 \%)$.

Values of porosity range from 0.0 to 11.3 per cent, with almost all of the samples (83) having values less than 6.0 per cent. As expected at low confining pressures, the high-density, high-velocity samples (notably those from the lower sections of Hole 418A) exhibit low porosities. What is significant is the fact that the velocity/porosity correlation is not substantially reduced at $6 \mathrm{kbar}$, as might be anticipated if the confining pressure were effective in closing the cracks and voids in the basalt which are responsible for its porosity. An attempt to correlate the increase in measured velocity with sample porosity in the 0.2 to $1.0 \mathrm{kbar}$ and 0.2 to $6.0 \mathrm{kbar}$ intervals shows the increase in velocity to be almost totally independent of porosity, the correlation coefficients being on the order of 0.10 to 0.20 .

The independence of the velocity/porosity relationship from confining pressure may be a result of the mechanism by which voids are formed in oceanic basalts. Plutonic rocks which have been exposed at the earth's surface were formed in regimes of high confining pressure. A large part of the porosity in such samples can probably be related to the effects of pressure release; conversely, increasing confining pressure during measurements made on such rocks should tend to reverse these effects. This is not the case for oceanic basalts which were extruded in a molten state at ocean ridge crests where the confining pressure due to the overlying column of sea water is generally on the order of only 0.25 to $0.35 \mathrm{kbar}$. Voids formed in this regime can be expected to be either vesicles due to trapped magmatic gases or cracks which are the result of thermal contraction as the basalt cools. Neither of these void types need close completely under the application of a high confining pressure. Furthermore, the effect of alteration of the basalts at the ridge crests might further tend to inhibit the closure of cracks at high confining pressures. Since the cracks responsible (in part) for the porosity of submarine basalts were formed by thermal contraction rather than pressure release, it would be interesting, especially in the fresher samples, to examine the effect of elevated temperatures on the velocity/porosity relationship.

\section{DENSITY/POROSITY RELATIONSHIPS}

The plot of density versus porosity (Figure 3 ) exhibits a trend which, as expected, indicates a lower bulk density for the samples with higher porosity. The large amount of scatter about the regression line in comparison to the velocity/density and velocity/porosity comparisons in Figures 1 and 2 is probably due both to the low accuracy of the porosity measurement and to the extent and mineralogy of alteration, which would tend to lower density values independently of porosity.

\section{ACKNOWLEDGMENTS}

J. Hull, G. Bussod, and R. Prior assisted in the velocity measurements. Financial support was provided by the Office of Naval Research Contract N-00014-75-C-0502.

\section{REFERENCES}

Birch, F., 1960. The velocity of compressional waves in rocks to 10 kilobars, 1, J. Geophys. Res., v. 65, p. 1083-1102. 
TABLE 2

Regression Line Parameters

\begin{tabular}{|c|c|c|c|c|c|c|}
\hline $\mathrm{Vp}=\mathrm{a}+\mathrm{b} \rho$ & & & & & & \\
\hline $\begin{array}{l}\text { Pressure, } \\
\text { kbar }\end{array}$ & $\mathrm{N}$ & $\underset{(\mathrm{km} / \mathrm{s})}{\mathrm{a}}$ & $\left(\mathrm{km} / \mathrm{s}^{\mathrm{b}} / \mathrm{g} / \mathrm{cm}^{3}\right)$ & $\begin{array}{l}\mathrm{S}_{(\mathrm{V}, \rho)} \\
(\mathrm{km} / \mathrm{s})\end{array}$ & r & $\begin{array}{l}\mathrm{r}^{2} \\
(\%)\end{array}$ \\
\hline 0.2 & 88 & -5.909 & 4.146 & 0.153 & 0.95 & 91 \\
\hline 1.0 & 88 & -5.272 & 3.968 & 0.155 & 0.95 & 90 \\
\hline 6.0 & 88 & -4.658 & 3.853 & 0.168 & 0.94 & 87 \\
\hline \multicolumn{7}{|l|}{$\mathrm{Vp}=\mathrm{a}+\mathrm{b} \phi$} \\
\hline $\begin{array}{l}\text { Pressure, } \\
\text { kbar }\end{array}$ & $\mathrm{N}$ & $\underset{(\mathrm{km} / \mathrm{s})}{\mathrm{a}}$ & $\begin{array}{c}\mathrm{b} \\
(\mathrm{km} / \mathrm{s} / \%)\end{array}$ & $\begin{array}{l}\mathrm{S}_{(\mathrm{V}, \phi)} \\
(\mathrm{km} / \mathrm{s})\end{array}$ & I & $\begin{array}{l}\mathrm{r}^{2} \\
(\%)\end{array}$ \\
\hline 0.2 & 85 & 6.238 & -0.222 & 0.233 & 0.89 & 78 \\
\hline 1.0 & 85 & 6.349 & -0.212 & 0.234 & 0.88 & 77 \\
\hline 6.0 & 85 & 6.624 & -0.205 & 0.241 & 0.86 & 74 \\
\hline \multicolumn{7}{|l|}{$\rho=\mathrm{a}+\mathrm{b} \phi$} \\
\hline & $\mathrm{N}$ & $\stackrel{\mathrm{a}}{\left(\mathrm{g} / \mathrm{cm}^{3}\right)}$ & $\left(\mathrm{g} / \mathrm{cm}^{3} / \%\right)$ & $\begin{array}{c}\mathrm{S}_{(\rho, \phi)} \\
\left(\mathrm{g} / \mathrm{cm}^{3}\right)\end{array}$ & $\mathrm{r}$ & $\begin{array}{l}\mathrm{r}^{2} \\
(\%)\end{array}$ \\
\hline & 85 & 2.918 & -0.049 & 0.061 & 0.85 & 72 \\
\hline \multicolumn{7}{|l|}{$\phi=a+b \rho$} \\
\hline & $\mathrm{N}$ & $\begin{array}{c}\mathrm{a} \\
(\%)\end{array}$ & $\begin{array}{c}\mathrm{b} \\
\left(\% /\left(\mathrm{g} / \mathrm{cm}^{3}\right)\right)\end{array}$ & $\begin{array}{c}\mathrm{S}_{(\phi \rho)} \\
(\%)\end{array}$ & t & $\begin{array}{l}r^{2} \\
(\%)\end{array}$ \\
\hline & 85 & 43.64 & -14.72 & 1.060 & 0.85 & 72 \\
\hline$\rho=a+b V p$ & & & & & & \\
\hline $\begin{array}{l}\text { Pressure, } \\
\text { kbar }\end{array}$ & $\mathrm{N}$ & $\underset{\left(\mathrm{g} / \mathrm{cm}^{3}\right)}{\mathrm{a}}$ & $\stackrel{\mathrm{b}^{\mathrm{b}}}{\left(\mathrm{g} / \mathrm{cm}^{3} / \mathrm{km} / \mathrm{s}\right)}$ & $\begin{array}{l}(\rho, V p) \\
\left(\mathrm{g} / \mathrm{cm}^{3}\right)\end{array}$ & $\mathrm{r}$ & $\begin{array}{l}\mathrm{r}^{2} \\
(\%)\end{array}$ \\
\hline 0.2 & 88 & 1.552 & 0.219 & 0.035 & 0.95 & 91 \\
\hline 1.0 & 88 & 1.479 & 0.226 & 0.037 & 0.95 & 90 \\
\hline 6.0 & 88 & 1.407 & 0.227 & 0.041 & 0.94 & 87 \\
\hline \multicolumn{7}{|l|}{$\phi=a+b V p$} \\
\hline $\begin{array}{c}\text { Pressure, } \\
\text { kbar }\end{array}$ & $\mathrm{N}$ & $\begin{array}{c}a \\
(\%)\end{array}$ & $\begin{array}{c}\mathrm{b} \\
\% /(\mathrm{km} / \mathrm{s})\end{array}$ & $\begin{array}{c}\mathrm{S}_{(\phi, \mathrm{V} p)} \\
(\%)\end{array}$ & I & $\begin{array}{l}\mathrm{r}^{2} \\
(\%)\end{array}$ \\
\hline 0.2 & 85 & 22.52 & -3.524 & 0.929 & 0.89 & 78 \\
\hline 1.0 & 85 & 23.57 & -3.621 & 0.966 & 0.88 & 77 \\
\hline 6.0 & 85 & 24.65 & -3.624 & 1.020 & 0.86 & 74 \\
\hline
\end{tabular}

Note: $\mathrm{N}$ is the number of data points; $\mathrm{S}(\mathrm{a}, \mathrm{b})$, standard error of estimate of $b$ on $a ; r$, correlation coefficient; $\mathrm{r}^{2}$, coefficient of determination; $\rho$, wet bulk density; $\phi$, porosity; $V_{\mathrm{p}}$, compressional wave velocity.

Christensen, N.I. and Salisbury, M.H., 1975. Structure and constitution of the lower oceanic crust, Rev. Geophys. Space Phys., v. 13, p. 57-86.
Christensen, N.I. and Shaw, G.H., 1970. Elasticity of mafic rocks from the Mid-Atlantic Ridge, Geophys. J. Roy. Astron. Soc., v. 20 , p. $271-284$. 


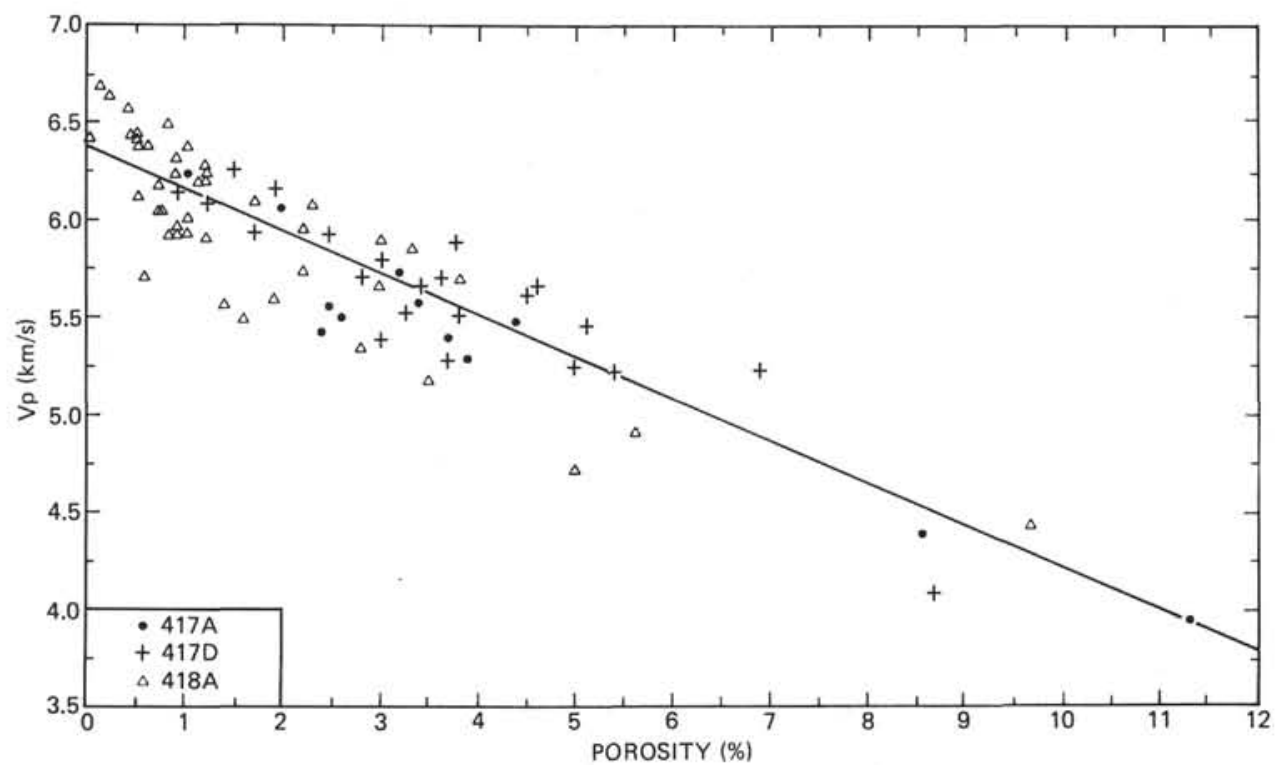

Figure 2. Compressional wave velocity at 1.0 kbar plotted against effective porosity. Linear solution from Table 1.

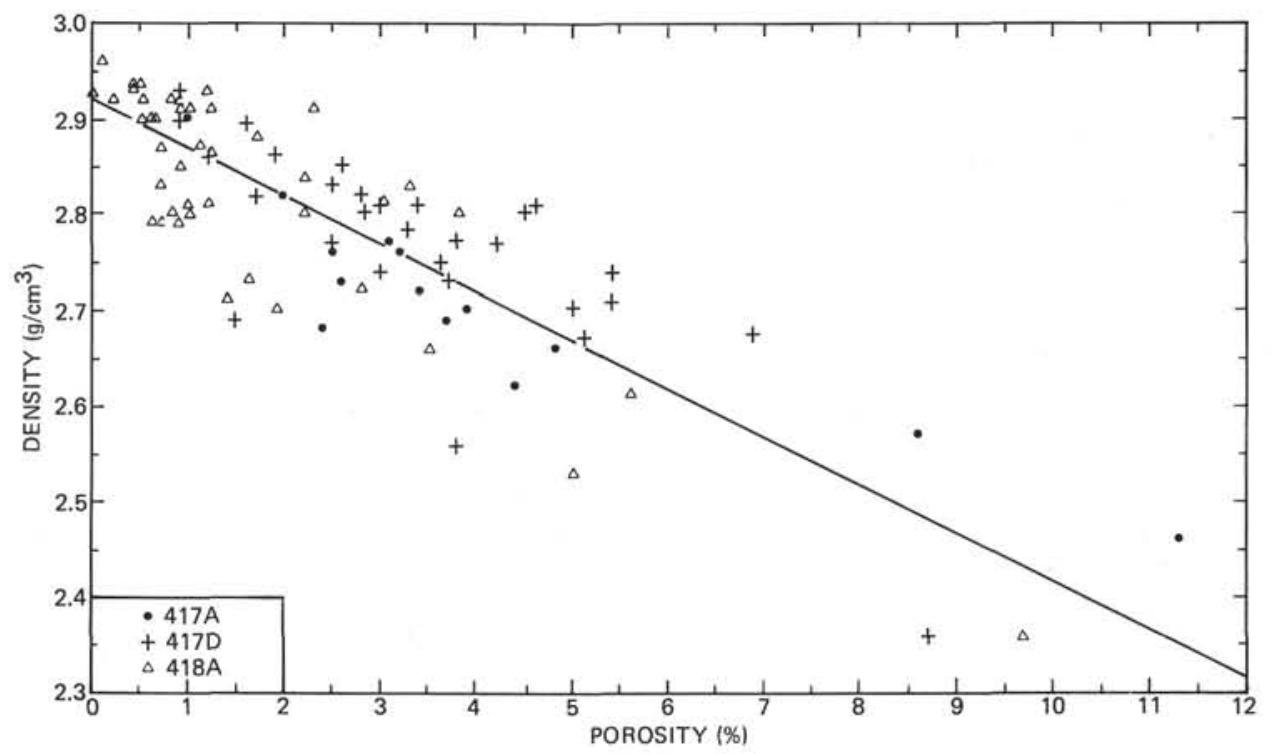

Figure 3. Wet-bulk density plotted against porosity. Linear solution from Table 1. 